\title{
Statistical Optimality of Hermite Splines
}

\author{
Virginie Uhlmann, Julien Fageot, Harshit Gupta, Michael Unser \\ Biomedical Imaging Group \\ École polytechnique fédérale de Lausanne (EPFL) \\ Switzerland \\ Email: virginie.uhlmann@epfl.ch, julien.fageot@epfl.ch,g.harshit@iitg.ernet.in, michael.unser@epfl.ch
}

\begin{abstract}
Hermite splines are commonly used for interpolating data when samples of the derivative are available, in a scheme called Hermite interpolation. Assuming a suitable statistical model, we demonstrate that this method is actually optimal for reconstructing random signals in Papoulis' generalized sampling framework. We focus on second-order Lévy processes-the integrated version of Lévy processes-and rely on cubic Hermite splines to approximate the original continuous-time signal from its samples and its derivatives at integer values. We statistically justify the use of this reconstruction scheme by demonstrating the equivalence between cubic Hermite interpolation and the linear minimum mean-square error (LMMSE) estimation of a secondorder Lévy process. We finally illustrate the cubic Hermite reconstruction scheme on an example of a discrete sequence sampled from the realization of a stochastic process.
\end{abstract}

\section{INTRODUCTION}

Reconstruction using interpolation plays an essential role in communications and signal processing as it creates a bridge between sampled and analog signals. Foundations of interpolation are attributed to Shannon [1], who demonstrated that perfect reconstruction was possible for sampled bandlimited signals. In its initial formulation, Shannon's theory involves uniform samples of the original continuous-time signal. Papoulis [2] proposed an extension of this framework referred to as generalized sampling. The underlying idea is that a signal can be fully characterized, and thus reconstructed, with many different kind of data, uniform samples being only one of them. These initial formulations of the sampling problem were designed for bandlimited signals and involved ideal filters, making them mostly unusable in practice although theoretically beautiful.

Extensions without band limited constraints relying on spline-based signal-representation models have been proposed [3]. For instance, the well-known Hermite interpolation setting proposed by Schoenberg [4] is a typical instance of Papoulis' generalization without band limited constraints. In Hermite interpolation, a continuously differentiable function is generated from two discrete sequences that correspond to samples of a function and its derivative, respectively, and the data are fitted using Hermite-spline basis functions. Following the notation of [3], Hermite interpolation is obtained by choosing $h_{1}(t)=\delta(t)$ and $h_{2}(t)=\delta^{\prime}(t)$ as analysis filters, where $\delta$ is the Dirac delta function.

In the generalized sampling framework, reconstruction is based on variational principles [5]. However, a statistical

978-1-4673-7353-1/15/\$31.00 (C)2015 IEEE interpretation can be given to these deterministic spline-fitting methods through the minimal mean square-error (MMSE) criterion, extending their use to the optimal reconstruction of random processes in the least-squares sense. In the stationary case, the generalized spline interpolator was demonstrated [6] to be the linear minimum mean-square error (LMMSE) estimator of the continuous-time stochastic process $s(t), t \in \mathbb{R}$,

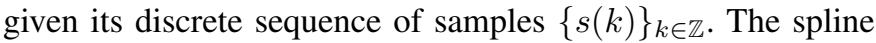
interpolation algorithm is therefore optimal for the estimation of a wide family of stationary random signals. Similar results have been demonstrated by [7], [8]: the optimal (MMSE) interpolator for first-order Lévy processes is the piecewise linear spline. Note that these processes, defined as $s$ such that $\mathrm{D} s=w$, are non-stationary but (wide-sense) self-similar.

We aim at extending these results to the Hermite-spline interpolation framework, relying on the two sequences of samples $\left\{s(k), s^{\prime}(k)\right\}_{k \in \mathbb{Z}}$. To do so, we consider self-similar second-order Lévy processes $s$ such that $\mathrm{D}^{2} s=w$, for which the derivative exists. We first introduce the notations and objects related to the cubic Hermite-interpolation problem as formulated by Schoenberg and describe the second-order Lévy processes, which are relevant to this work. Then, we present our main contribution, namely, the statistical optimality of the cubic Hermite spline-reconstruction method for the selfsimilar stochastic processes we consider. We give a formal proof of this optimality and finally propose an illustrative example where we apply the proposed approach to samples of a second-order Gaussian process.

\section{THEORETICAL BACKGROUND}

Through the paper, we shall often use the notation $f(t)$ for

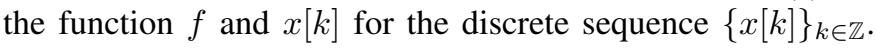
In addition, the sequence of samples of a function $f(t)$ at the integers is denoted by $f(k)=\left.f(t)\right|_{t=k}$.

\section{A. Hermite Spline Interpolation}

Schoenberg [4], [9] defines the cardinal cubic Hermite interpolation problem as follows: Knowing the discrete sequences of numbers $c[k]$ and $d[k], k \in \mathbb{Z}$, we look for a continuous function $f(t), t \in \mathbb{R}$, satisfying $f(k)=c[k], f^{\prime}(k)=d[k]$ for all $k \in \mathbb{Z}$, and such that $f$ belongs to $S_{4,2}$. We denote by $S_{4,2}$ the class of spline functions of degree 3 with knots of multiplicity 2 at the integers. The existence and uniqueness of the solution is guaranteed [4] provided that $c[k]$ and $d[k]$ are in $S^{\prime}(\mathbb{Z})$, the space of functions of slow growth. We say that 


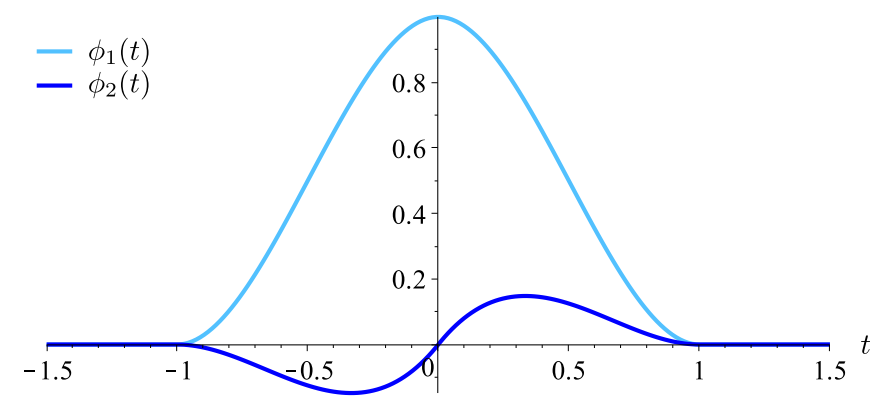

(a)

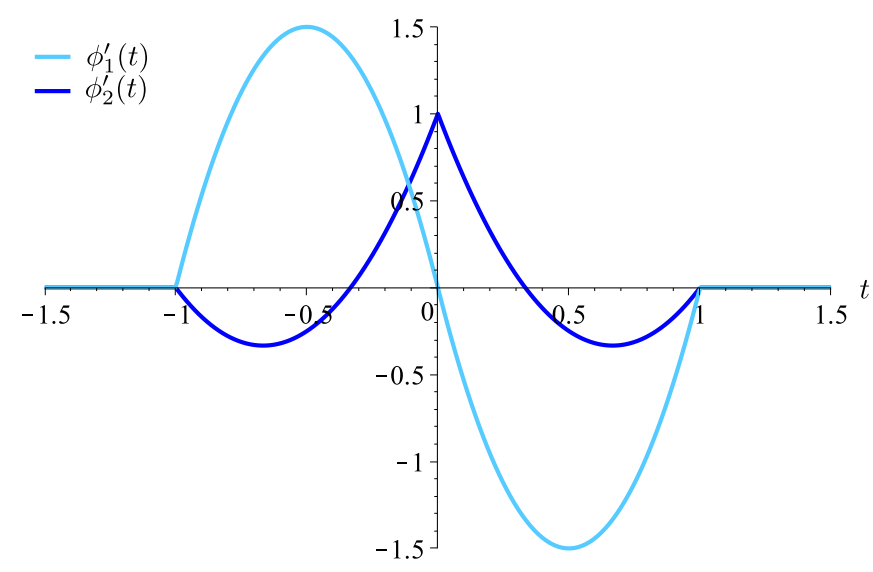

(b)

Fig. 1. The cubic Hermite splines (a) $\phi_{1}$ and $\phi_{2}$ and (b) their first derivatives. The two functions and their derivatives are vanishing at the integers with the exception of $\phi_{1}(0)=1$ and $\phi_{2}^{\prime}(0)=1$ (interpolation condition). Their support is of size two.

a sequence $x[k]$ is in $S^{\prime}(\mathbb{Z})$ if there exist two real constants $N$ and $C$ such that $|x[k]| \leq C\left(|k|^{N}+1\right)$. In addition, the spline function $f_{\mathrm{Her}}$, which is the unique solution of the second-order cardinal Hermite interpolation problem, is explicitly given [4] by

$$
f_{\text {Her }}(t)=\sum_{k \in \mathbb{Z}}\left(c[k] \phi_{1}(t-k)+d[k] \phi_{2}(t-k)\right) .
$$

The explicit expressions of the two Hermite cubic splines $\phi_{1}, \phi_{2}$ are found in [9], [10] as

$$
\begin{aligned}
& \phi_{1}(t)= \begin{cases}(2|t|+1)(|t|-1)^{2} & \text { for } 0 \leq|t| \leq 1 \\
0 & \text { for }|t|>1,\end{cases} \\
& \phi_{2}(t)= \begin{cases}t(|t|-1)^{2} & \text { for } 0 \leq|t| \leq 1 \\
0 & \text { for }|t|>1 .\end{cases}
\end{aligned}
$$

These two functions are represented in Figure 1.

In addition to the restriction of the support to the interval $[-1,1]$, the main property of this construction is that the two generators $\phi_{1}, \phi_{2}$ and their derivatives $\phi_{1}^{\prime}, \phi_{2}^{\prime}$ satisfy the joint interpolation conditions

$$
\phi_{1}(k)=\delta[k], \quad \phi_{2}^{\prime}(k)=\delta[k], \quad \phi_{1}^{\prime}(k)=0, \quad \phi_{2}(k)=0,
$$

for all $k \in \mathbb{Z}$, where $\delta[k]$ is the discrete unit impulse. In this setting, the function $\phi_{1}(t)$ interpolates the point values $c[k]$ while the first derivative of $\phi_{2}(t)$ interpolates the derivative values $d[k]$.

The Hermite-spline space of functions

$$
\left\{\sum_{k \in \mathbb{Z}}\left(c[k] \phi_{1}(\cdot-k)+d[k] \phi_{2}(\cdot-k)\right): c[k], d[k] \in S^{\prime}(\mathbb{Z})\right\}
$$

can be seen as a space of cubic splines that can accommodate quadratic transitions, or, in other words, of splines of degree 3 with regularity $C^{1}$ at the knots. Note that there is no conceptual difficulty in considering $c[k], d[k] \in S^{\prime}(\mathbb{Z})$ rather than the condition $c[k], d[k] \in l_{2}(\mathbb{Z})$ that is more commonly found in the literature, since the Hermite basis functions $\phi_{1}$ and $\phi_{2}$ are compactly supported.

\section{B. Second-Order Lévy Processes}

We consider continuous-time random processes $s$ solutions of the stochastic differential equation

$$
\mathrm{D}^{2} s=w,
$$

where $\mathrm{D}$ is the differential operator and $w$ a Lévy white noise with finite variance and zero mean $\mathbb{E}\{s(t)\}=0$ for all $t \in \mathbb{R}$, with boundary conditions $s(0)=s^{\prime}(0)=0$. The process $s$ is called a second-order Lévy process [11]. Being the integration of a Lévy process, second-order Lévy processes are smoother. Such processes include the integrated version of a Brownian motion, which corresponds to a Gaussian white noise (Figures $2 \mathrm{a}$ and $2 \mathrm{~b}$ ). They can as well be driven by compound-Poisson white noise (Figure 2c), which yield a piecewise linear process, as illustrated in Figure 2d. The second-order Lévy process $s$ is continuous and its derivative $s^{\prime}$ is a first-order Lévy process [12]. The samples of $s$ and $s^{\prime}$ at the integers are therefore well-defined. As an example, the Poisson process is not differentiable at the jumps locations. However, the intersection of the set of transitions and the sampling locations (i.e., the integers) is empty with probability 1. A throughout mathematical formalization of these objects can be found in [11], [13].

Let $c_{s s}(t, \tau)=\mathbb{E}\{s(t) s(\tau)\}$ be the autocorrelation function of $s$. We note that the second-order Lévy process $s$ is widesense self-similar with scaling order $H=\frac{3}{2}$, meaning that $s$ and $a^{\frac{3}{2}} s(\dot{\bar{a}})$ have the same second-order statistics $\forall a>0$. Equivalently, its autocorrelation function satisfies $c_{s s}(t, \tau)=$ $a^{3} c_{s s}\left(\frac{t}{a}, \frac{\tau}{a}\right)$. In the Gaussian case, $s$ is even strongly selfsimilar, which means that $s$ and $a^{\frac{3}{2}} s(\dot{\dot{a}})$ have the same law. Moreover, $s$ is non-stationary but has second-order stationary increments [11]. The explicit expression of the autocorrelation function $c_{s s}$ is given as [14]

$$
c_{s s}(t, \tau)=\frac{\sigma^{2}}{4}\left(|t-\tau|^{3}-|t|^{3}-|\tau|^{3}+3 t \tau(|t|+|\tau|)\right)
$$

and is normalized such that $\operatorname{Var}\{s(t)\}=\sigma^{2}|t|^{3}$.

Our goal is to study the estimation of $s$ at a fixed time point $t_{0}$ given $\left\{s(k), s^{\prime}(k)\right\}_{k \in \mathbb{Z}}$. Since the process $s$ is in $S^{\prime}(\mathbb{R})$ [15], it thus fits the framework of II-A. Hence, the function $t \mapsto$ $c_{s s}\left(t, t_{0}\right)$ and its derivatives can be expanded in a Hermite basis. 


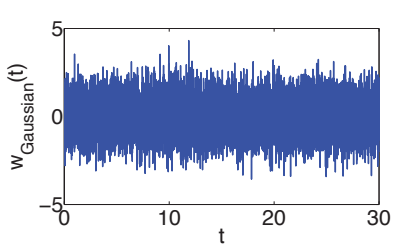

(a)

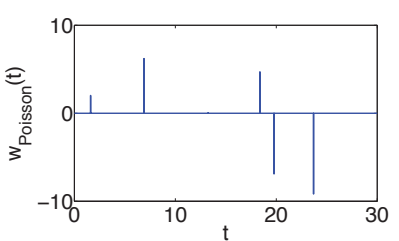

(c)

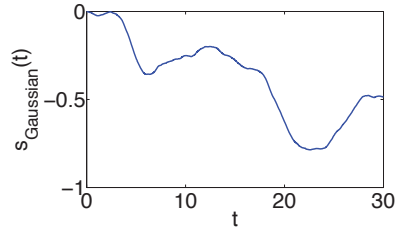

(b)

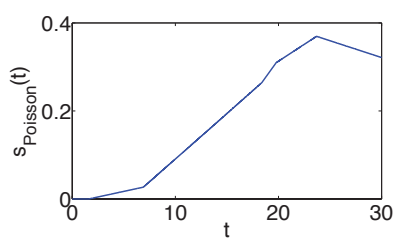

(d)
Fig. 2. Second-order Lévy processes for different types of noise. (a) Randomly generated Gaussian noise and (b) corresponding second-order Gaussian process. (c) Randomly generated Poisson noise with normally distributed jumps and (d) corresponding second-order Poisson process. We use the conventional way of representing white noises although they are not defined pointwise.

\section{MMSE ESTIMATION AND INTERPOLATION}

We here state and prove our main result about the statistical optimality of Hermite spline reconstruction.

For a fixed $t_{0}$, we aim at determining a linear estimator of $s\left(t_{0}\right)$ given the random sequences $\left\{s(k), s^{\prime}(k)\right\}_{k \in \mathbb{Z}}$. The estimator will therefore be a random variable of the form

$$
\tilde{s}\left(t_{0}\right)=\sum_{k \in \mathbb{Z}}\left(a_{t_{0}}[k] s(k)+b_{t_{0}}[k] s^{\prime}(k)\right),
$$

where $a_{t_{0}}[k]$ and $b_{t_{0}}[k]$ are two infinite deterministic sequences of regression coefficients. The linear minimum meansquare error (LMMSE) estimator $\tilde{s}_{\text {LMMSE }}\left(t_{0}\right)$ corresponds to the estimator $\tilde{s}\left(t_{0}\right)$ that minimizes the mean-square error $\mathbb{E}\left\{\left|s\left(t_{0}\right)-\tilde{s}\left(t_{0}\right)\right|^{2}\right\}$.

The random sequences $s(k)$ and $s^{\prime}(k)$ are in $S^{\prime}(\mathbb{Z})$ almost surely [11]. We therefore make sure that the summation in (2) is well-defined by restricting ourselves to sequences $a_{t_{0}}[k], b_{t_{0}}[k] \in S(\mathbb{Z})$, the space of sequences that decay faster than any polynomial.

Theorem 1. Let $s$ be such that $\mathrm{D}^{2} s=w$. Then, the linear minimum mean-square error (LMMSE) estimators of $s(t)$ and $s^{\prime}(t)$ at $t=t_{0}$ given the samples $\left\{s(k), s^{\prime}(k)\right\}_{k \in \mathbb{Z}}$ are

$$
\begin{aligned}
\tilde{s}_{\text {LMMSE }}\left(t_{0}\right) & =s_{\text {Her }}\left(t_{0}\right) \\
\widetilde{s}_{\text {LMMSE }}^{\prime}\left(t_{0}\right) & =s_{\text {Her }}^{\prime}\left(t_{0}\right),
\end{aligned}
$$

where

$$
s_{\mathrm{Her}}(t)=\sum_{k \in \mathbb{Z}}\left(s(k) \phi_{1}\left(t_{0}-k\right)+s^{\prime}(k) \phi_{2}\left(t_{0}-k\right)\right)
$$

is the Hermite interpolation of $\left\{s(k), s^{\prime}(k)\right\}_{k \in \mathbb{Z}}$.

Proof. Note that, since boundary conditions impose that $s(0)=s^{\prime}(0)=0$, we exclude the index $k=0$ through the proof for convenience purpose.
Applying the orthogonality principle [16] yields the two equations

$$
\begin{aligned}
\mathbb{E}\left\{s(n)\left(s\left(t_{0}\right)-\tilde{s}_{\text {LMMSE }}\left(t_{0}\right)\right)\right\} & =0, \\
\mathbb{E}\left\{s^{\prime}(n)\left(s\left(t_{0}\right)-\tilde{s}_{\text {LMMSE }}\left(t_{0}\right)\right)\right\} & =0 .
\end{aligned}
$$

By definition of the autocorrelation function, we have that

$$
\begin{aligned}
\mathbb{E}\{s(t) s(\tau)\} & =c_{s s}(t, \tau)=c_{s s}(\tau, t), \\
\mathbb{E}\left\{s^{\prime}(t) s(\tau)\right\} & =\partial_{1} c_{s s}(t, \tau), \\
\mathbb{E}\left\{s(t) s^{\prime}(\tau)\right\} & =\partial_{2} c_{s s}(t, \tau)=\partial_{1} c_{s s}(\tau, t) .
\end{aligned}
$$

Rearranging (3) and plugging in (2), we obtain

$$
\begin{aligned}
\mathbb{E}\left\{s(n) s\left(t_{0}\right)\right\} & =\mathbb{E}\left\{s(n) \tilde{s}_{\text {LMMSE }}\left(t_{0}\right)\right\} \\
& =\sum_{k \in \mathbb{Z} \backslash\{0\}}\left(a_{t_{0}}[k] \mathbb{E}\{s(n) s(k)\}\right. \\
& \left.\quad+b_{t_{0}}[k] \mathbb{E}\left\{s(n) s^{\prime}(k)\right\}\right)
\end{aligned}
$$

which, from (5) and (6), can be rewritten as

$$
c_{s s}\left(n, t_{0}\right)=\sum_{k \in \mathbb{Z} \backslash\{0\}}\left(a_{t_{0}}[k] c_{s s}(n, k)+b_{t_{0}}[k] \partial_{2} c_{s s}(n, k)\right) \text {. }
$$

Doing similar operations with (4), we obtain the system

$$
\left\{\begin{array}{l}
c_{s s}\left(n, t_{0}\right)=\sum_{k \in \mathbb{Z} \backslash\{0\}}\left(a_{t_{0}}[k] c_{s s}(n, k)+b_{t_{0}}[k] \partial_{2} c_{s s}(n, k)\right) \\
\partial_{1} c_{s s}\left(n, t_{0}\right)=\sum_{k \in \mathbb{Z} \backslash\{0\}}\left(a_{t_{0}}[k] \partial_{1} c_{s s}(n, k)+b_{t_{0}}[k] \partial_{2} \partial_{1} c_{s s}(n, k)\right) .
\end{array}\right.
$$

As $c_{s s}(n, \cdot)$ and $\partial_{1} c_{s s}(n, \cdot)$ belong to the space spanned by the cubic Hermite splines $\phi_{1}$ and $\phi_{2}$, we can expand them following (1) as

$$
\begin{aligned}
& c_{s s}(n, \cdot)=\sum_{k \in \mathbb{Z} \backslash\{0\}}\left(c_{s s}(n, k) \phi_{1}(\cdot-k)+\partial_{2} c_{s s}(n, k) \phi_{2}(\cdot-k)\right), \\
& \partial_{1} c_{s s}(n, \cdot)=\sum_{k \in \mathbb{Z} \backslash\{0\}}\left(\partial_{1} c_{s s}(n, k) \phi_{1}(\cdot-k)+\partial_{2} \partial_{1} c_{s s}(n, k) \phi_{2}(\cdot-k)\right) .
\end{aligned}
$$

The system to solve thus becomes

$$
\left\{\begin{array}{c}
\sum_{k \in \mathbb{Z} \backslash\{0\}}\left(c_{s s}(n, k) \phi_{1}\left(t_{0}-k\right)+\partial_{2} c_{s s}(n, k) \phi_{2}\left(t_{0}-k\right)\right) \\
=\sum_{k \in \mathbb{Z} \backslash\{0\}}\left(a_{t_{0}}[k] c_{s s}(n, k)+b_{t_{0}}[k] \partial_{2} c_{s s}(n, k)\right) \\
\sum_{k \in \mathbb{Z} \backslash\{0\}}\left(\partial_{1} c_{s s}(n, k) \phi_{1}\left(t_{0}-k\right)+\partial_{2} \partial_{1} c_{s s}(n, k) \phi_{2}\left(t_{0}-k\right)\right) \\
=\sum_{k \in \mathbb{Z} \backslash\{0\}}\left(a_{t_{0}}[k] \partial_{1} c_{s s}(n, k)+b_{t_{0}}[k] \partial_{2} \partial_{1} c_{s s}(n, k)\right) .
\end{array}\right.
$$

Let the sequences $u$ and $v$ be such that $u[k]=\phi_{1}\left(t_{0}-k\right)-$ $a_{t_{0}}[k]$ and $v[k]=\phi_{2}\left(t_{0}-k\right)-b_{t_{0}}[k], k \in \mathbb{Z} \backslash\{0\}$. The system (7) can be reformulated in blocs matrix notation as

$$
\left[\begin{array}{ll}
\mathrm{A} & \mathrm{B} \\
\mathrm{B}^{T} & \mathrm{C}
\end{array}\right]\left[\begin{array}{l}
u \\
v
\end{array}\right]=\left[\begin{array}{l}
0 \\
0
\end{array}\right],
$$

where $\mathrm{A}[n, k]=c_{s s}(n, k), \mathrm{B}[n, k]=\partial_{2} c_{s s}(n, k), \mathrm{B}^{T}[n, k]=$ $\partial_{1} c_{s s}(n, k)$ (see (6)) and $\mathrm{C}[n, k]=\partial_{1} \partial_{2} c_{s s}(n, k)$, with a 
slight abuse of notation due to the fact that these sequences and matrices are bi-infinite. To finish the proof, we observe that

$$
\begin{aligned}
{\left[\begin{array}{l}
u \\
v
\end{array}\right]^{\mathrm{T}}\left[\begin{array}{ll}
\mathrm{A} & \mathrm{B} \\
\mathrm{B}^{T} & \mathrm{C}
\end{array}\right]\left[\begin{array}{l}
u \\
v
\end{array}\right] } & =\operatorname{Var}\left\{\sum_{k \in \mathbb{Z} \backslash\{0\}}\left(u[k] s(k)+v[k] s^{\prime}(k)\right)\right\} \\
& =0,
\end{aligned}
$$

which implies that $\sum_{k \in \mathbb{Z} \backslash\{0\}}\left(u[k] s(k)+v[k] s^{\prime}(k)\right)=0$ almost surely. The only solution is therefore $u[k]=v[k]=0$ for all $k \in \mathbb{Z} \backslash\{0\}$. Indeed, if one of the $u[k]$ or $v[k]$ is nonzero, say for instance $u\left[k_{0}\right]$, it implies that $s\left(k_{0}\right)$ can be deterministically evaluated from the values of all the $s(k)$ and $s^{\prime}(k)$ except $s\left(k_{0}\right)$, which is absurd. Therefore,

$$
\begin{aligned}
a_{t_{0}}[k] & =\phi_{1}\left(t_{0}-k\right), \\
b_{t_{0}}[k] & =\phi_{2}\left(t_{0}-k\right),
\end{aligned}
$$

and the LMMSE is finally given by

$$
\begin{aligned}
\tilde{s}_{\mathrm{LMMSE}}\left(t_{0}\right) & =s_{\mathrm{Her}}\left(t_{0}\right) \\
& =\sum_{k \in \mathbb{Z}}\left(s(k) \phi_{1}\left(t_{0}-k\right)+s^{\prime}(k) \phi_{2}\left(t_{0}-k\right)\right) .
\end{aligned}
$$

The proof for the problem of estimating $s^{\prime}\left(t_{0}\right)$ using $\left\{s(k), s^{\prime}(k)\right\}_{k \in \mathbb{Z}}$ is obtained with a similar development starting from

$$
\begin{aligned}
\mathbb{E}\left\{s(n)\left(s^{\prime}\left(t_{0}\right)-\widetilde{s}^{\prime}{ }_{\text {LMMSE }}\left(t_{0}\right)\right)\right\} & =0, \\
\mathbb{E}\left\{s^{\prime}(n)\left(s^{\prime}\left(t_{0}\right)-\widetilde{s}_{\text {LMMSE }}^{\prime}\left(t_{0}\right)\right)\right\} & =0,
\end{aligned}
$$

and the LMMSE is given by

$$
\begin{aligned}
{\widetilde{s^{\prime}}}_{\text {LMMSE }}\left(t_{0}\right) & =s_{\mathrm{Her}}^{\prime}\left(t_{0}\right) \\
& =\sum_{k \in \mathbb{Z}}\left(s(k) \phi_{1}^{\prime}\left(t_{0}-k\right)+s^{\prime}(k) \phi_{2}^{\prime}\left(t_{0}-k\right)\right) .
\end{aligned}
$$

In the Gaussian case, it can be shown from Bayes theorem that the LMMSE is actually the MMSE [16], which leads to Proposition 1.

Proposition 1. If $w$ is Gaussian, then the Hermite interpolation of $\left\{s(k), s^{\prime}(k)\right\}_{k \in \mathbb{Z}}$ is also the MMSE of $w$.

Theorem 1 and Proposition 1 provide solid statistical ground for using Hermite spline interpolation in the case of secondorder Lévy processes. The novelty of these results is twofold. First, they rely on a sampling scheme involving both $s(k)$ and $s^{\prime}(k)$. Then, in addition to the optimal estimator of $s\left(t_{0}\right)$, they simultaneously give the optimal estimator of the derivative $s^{\prime}\left(t_{0}\right)$, which happens to be the derivative of the Hermite spline interpolator.

Our results exhibit strong links with [6], [7] as they also highlight the optimality of splines for the estimation of random processes from their samples. Our contribution however differs in two ways: first, from the fact that we know the samples $s^{\prime}(k)$ in addition to the $s(k)$, and then from the class of processes we study which are non-stationary (unlike [6], where the stationary case is investigated) and second-order (unlike [7], where first-order Lévy processes are considered).

We note that, in the Poisson case, our result implies that the best approximate of a piecewise linear (second-order Poisson) process is obtained by a piecewise cubic (Hermite) function. This result, which appears counter intuitive at first, can be explained as follows: On one hand, the second-order Poisson process is generated by the double integration of an impulsive noise, with non-uniformly distributed impulse locations. On the other hand, the Hermite interpolation scheme is cardinal with knots placed at the integers. In this setting, one cannot simply linearly interpolate between the "knots" of the secondorder Poisson process as they are not located at the integers.

\section{ILLUSTRATIVE EXAMPLES}

We illustrate the Hermite spline reconstruction from samples of a second-order Lévy process in the Gaussian case. A realization of a continuous-time random process $s$ such that $\mathrm{D}^{2} s=w$ and its continuous-time derivative $s^{\prime}$ are represented in Figures $3 \mathrm{a}$ and $3 \mathrm{~b}$, respectively. As, formally, $s=\mathrm{D}^{-2} w$, the process $s$ was generated by performing two rounds of integration on a Gaussian white noise while the derivative $s^{\prime}$ was obtained by integrating the noise once. The process and its derivative were sampled at the integers, yielding the two discrete sequences $s(k)$ and $s^{\prime}(k)$ represented in Figures $3 \mathrm{c}$ and $3 \mathrm{~d}$. Finally, the Hermite interpolation scheme (1) was applied to reconstruct the process $s$ from its samples $\left\{s(k), s^{\prime}(k)\right\}$, yielding the continuous-time $s_{\mathrm{Her}}$ displayed in Figure 3e. Similarly, the scheme was applied to reconstruct the continuously defined derivative $s^{\prime}$ from the samples $\left\{s(k), s^{\prime}(k)\right\}_{k \in \mathbb{Z}}$, yielding $s_{\text {Her }}^{\prime}$ shown in Figure 3f. The absolute reconstruction error $\left|s_{\mathrm{Her}}(t)-s(t)\right|$ is smaller than 0.01 (Figure $3 \mathrm{~g}$ ), and the absolute reconstruction error on the derivative $\left|s_{\text {Her }}^{\prime}(t)-s^{\prime}(t)\right|$ is smaller than 0.05 , as seen in Figure 3h.

\section{CONCLUSION}

In this paper, we demonstrated that Hermite spline reconstruction is the best linear estimator for the task of reconstructing a second-order Lévy process from its discrete samples. For the process $s$ satisfying $\mathrm{D}^{2} s=w$, Hermite spline reconstruction is even statistically optimal among all possible estimators when the underlying noise $w$ is Gaussian. We generated a realization of a second-order Gaussian process and used it to illustrate the sampling and reconstruction using Hermite splines. This example allowed us to highlight the small reconstruction error when using Hermite interpolation.

Results presented in this work hold for uniform samples. However, we expect that similar findings would be obtained in a non uniform sampling scheme, as it is already known in the case of reconstruction with mere function samples [8]. An extension of our problem would be to investigate the optimal estimation algorithm for continuous processes given collections of noise-corrupted measurements. We again expect to obtain results analogous to the ones presented for the ordinary sampling case in [6]. 


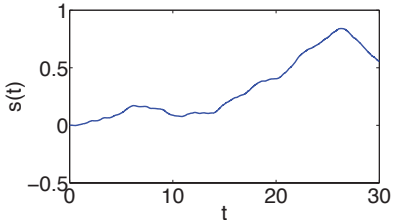

(a)

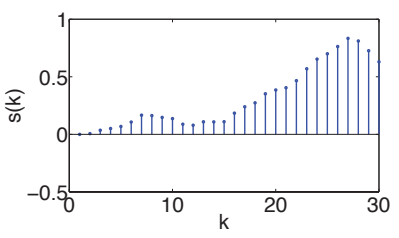

(c)

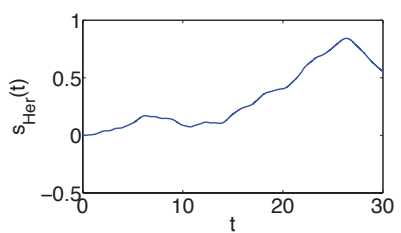

(e)

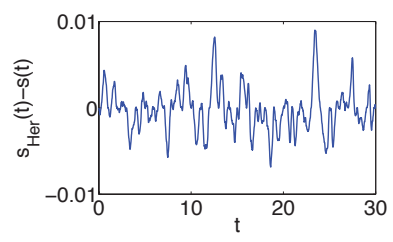

(g)

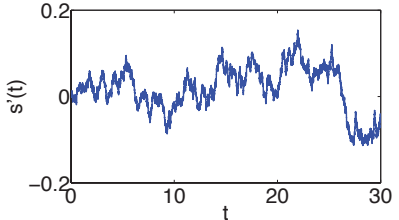

(b)

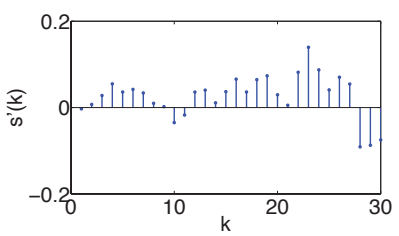

(d)

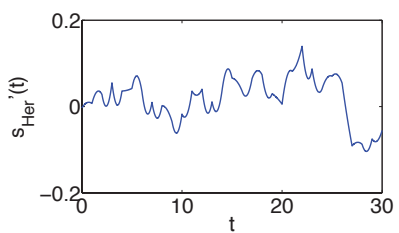

(f)

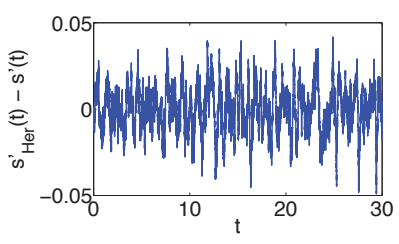

(h)
Fig. 3. Hermite spline reconstruction of a sampled second-order Gaussian process. (a) Realization of a second-order Gaussian process $s$ and (b) its continuous derivative $s^{\prime}$. (c) Discrete sequence of samples $s(k)$ drawn from $s(t)$ and (d) derivative sequence of samples $s^{\prime}(k)$ drawn from $s^{\prime}(t)$. (e) Reconstruction $s_{\mathrm{Her}}$ of the original continuous-time process $s$ using Hermite spline interpolation. (f) Reconstruction $s_{\text {Her }}^{\prime}$ of the original continuous-time derivative $s^{\prime}$ using Hermite spline interpolation. Reconstruction errors $(\mathrm{g})$ $\left(s_{\mathrm{Her}}(t)-s(t)\right)$ and (f) $\left(s_{\mathrm{Her}}^{\prime}(t)-s^{\prime}(t)\right)$.

\section{ACKNOWLEDGMENT}

This work is funded by the Swiss National Science Foundation under Grants 200020-144355 and 200020-121763 and the European Research Council under the European Union's Seventh Framework Programme (FP7/2007-2013) / ERC grant agreement $n^{\circ} 267439$.

\section{REFERENCES}

[1] M. Unser, "Sampling-50 Years after Shannon," Proceedings of the IEEE, vol. 88, no. 4, pp. 569-587, April 2000.

[2] A. Papoulis, "Generalized sampling expansion," IEEE Transactions on Circuits and Systems, vol. 24, no. 11, pp. 652-654, November 1977.

[3] M. Unser and J. Zerubia, "A generalized sampling theory without bandlimiting constraints," IEEE Transactions on Circuits and Systems-II: Analog and Digital Signal Processing, vol. 45, no. 8, pp. 959-969, August 1998.

[4] P. Lipow and I. Schoenberg, "Cardinal interpolation and spline functions III. Cardinal Hermite interpolation," Linear Algebra and its Applications, vol. 6, pp. 273-304, 1973.
[5] J. Kybic, T. Blu, and M. Unser, "Generalized sampling: A variational approach-Part I: Theory," IEEE Transactions on Signal Processing, vol. 50, no. 8, pp. 1965-1976, August 2002.

[6] M. Unser and T. Blu, "Generalized smoothing splines and the optimal discretization of the Wiener filter," IEEE Transactions on Signal Processing, vol. 53, no. 6, pp. 2146-2159, June 2005.

[7] A. Amini, P. Thévenaz, J. Ward, and M. Unser, "On the linearity of Bayesian interpolators for non-Gaussian continuous-time AR(1) processes," IEEE Transactions on Information Theory, vol. 59, no. 8, pp. 5063-5074, August 2013.

[8] A. Amini and M. Unser, "Optimal interpolation laws for stable AR(1) processes," in Proceedings of the Tenth International Workshop on Sampling Theory and Applications (SampTA'13), Bremen, Federal Republic of Germany, July 1-5, 2013, pp. 380-383.

[9] I. Schoenberg and A. Sharma, "Cardinal interpolation and spline functions V. The B-splines for cardinal Hermite interpolation," Linear Algebra and its Applications, vol. 7, no. 1, pp. 1-42, January 1973.

[10] W. Dahmen, B. Han, R.-Q. Jia, and A. Kunoth, "Biorthogonal multiwavelets on the interval: Cubic Hermite splines," Constructive Approximation, vol. 16, no. 2, pp. 221-259, February 2000.

[11] M. Unser and P. Tafti, "Sparse stochastic processes," in An Introduction to Sparse Stochastic Processes, 1st ed. Cambridge, United Kingdom: Cambridge University Press, August 2014, ch. 7, pp. 149-189.

[12] K. Sato, Lévy Processes and Infinitely Divisible Distributions, ser. Cambridge Studies in Advanced Mathematics. Cambridge, United Kingdom: Cambridge University Press, November 1999, vol. 68.

[13] M. Unser, P. Tafti, A. Amini, and H. Kirshner, "A unified formulation of Gaussian versus sparse stochastic processes-Part II: Discrete-domain theory," IEEE Transactions on Information Theory, vol. 60, no. 5, pp. 3036-3051, May 2014.

[14] T. Blu and M. Unser, "Self-similarity: Part II-Optimal estimation of fractal processes," IEEE Transactions on Signal Processing, vol. 55, no. 4, pp. 1364-1378, April 2007.

[15] M. Unser, P. Tafti, and Q. Sun, "A unified formulation of Gaussian versus sparse stochastic processes-Part I: Continuous-domain theory," IEEE Transactions on Information Theory, vol. 60, no. 3, pp. 19451962, March 2014.

[16] T. Moon and W. Stirling, Mathematical Methods and Algorithms for Signal Processing, 1st ed. Upper Saddle River, NJ, USA: Prentice Hall, August 1999. 